\title{
Anomalous diffusion in quantum Brownian motion with colored noise.
}

\author{
G. W. Ford \\ Department of Physics, University of Michigan,. Ann Arbor, MI 48109-1120 \\ R. F. O'Connell* \\ Department of Physics and Astronomy, \\ Louisiana State University, Baton Rouge, LA 70803-4001
}

(Dated: July 29, 2018)

\begin{abstract}
Anomalous diffusion is discussed in the context of quantum Brownian motion with colored noise. It is shown that earlier results follow simply and directly from the fluctuation-dissipation theorem. The limits on the long-time dependence of anomalous diffusion are shown to be a consequence of the second law of thermodynamics. The special case of an electron interacting with the radiation field is discussed in detail. We apply our results to wave-packet spreading.
\end{abstract}

PACS: 05.30.-d, 05.40.-a, 05.40.Jc

\footnotetext{
*E-mail: oconnell@phys.lsu.edu
} 


\section{INTRODUCTION}

There has been a recurrence of interest in the phenomenon of anomalous diffusion. We refer to a sampling of the literature [1, 2, 3, 4, 5, 6, 7, 8]. There have also appeared in the literature a variety of explanations for such behavior. In particular, Tsallis and others 4] have proposed that conventional Gibbs-Boltzmann statistics be generalized by extending the definition of entropy. On the other hand, it has been shown that an explanation using conventional statistics is possible if one uses a Fokker-Planck equation with either fractional derivatives [2] or time-dependent diffusion coefficients [7, 8]. In addition, a study using path integral methods [9] showed that anomalous diffusion appears in quantum Brownian motion with colored noise.

Our purpose here is first of all to show that these same results follow directly from the well known exact formula for the correlation function that can be obtained using the fluctuationdissipation theorem. In this section, we present general results which are applicable to an arbitrary heat bath. Then, in Secs. II to IV, we apply these results to the Ohmic heat bath, colored noise and a radiation heat bath (QED), respectively. In particularly, we note that the second law of thermodynamics places limits on the asymptotic behaviour. The physically realizable example of a charged particle coupled to the radiation field is given particular attention. In Sec. V, we apply our results to the spreading of a wave packet. Of special interest is the fact that the results in the QED case involve the bare mass. Finally, in Sec. VI, we present our conclusions.

Diffusion of a Brownian particle is characterized by the long time behavior of the mean square displacement, which for motion during time $t$ is defined to be

$$
s(t)=\left\langle[x(t)-x(0)]^{2}\right\rangle
$$

Normal diffusion corresponds to a linear time-dependence for long times, with the diffusion constant given by $D=\frac{1}{2} \lim _{t \rightarrow \infty} \dot{s}(t)$. Anomalous diffusion is characterized by a deviation from this linear time dependence of the form

$$
s(t) \sim t^{1+\gamma}, \quad-1<\gamma<1
$$

with $\gamma=0$ being the case of normal diffusion. Our discussion will be based on the familiar

formula for the correlation function, obtained using the fluctuation-dissipation theorem 10 , 
$11]$

$$
\frac{1}{2}\langle x(t) x(0)+x(0) x(t)\rangle=\frac{\hbar}{\pi} \int_{0}^{\infty} d \omega \operatorname{Im}\left\{\alpha\left(\omega+i 0^{+}\right)\right\} \operatorname{coth} \frac{\hbar \omega}{2 k T} \cos \omega t .
$$

Here $\alpha$ is the generalized susceptibility, which for a Brownian particle of mass $m$ is of the form

$$
\alpha(z)=\frac{1}{-m z^{2}-i z \tilde{\mu}(z)},
$$

where $\tilde{\mu}(z)$ is the Fourier transform of the memory function. For our purposes all we need know about $\tilde{\mu}(z)$ is that, as a consequence of the second law of thermodynamics, it must be what is termed a positive real function [12]. That is, $\tilde{\mu}(z)$ must be analytic with positive real part everywhere in the upper half plane and, in addition, its boundary value on the real axis, which in general may be a distribution, must satisfy the reality condition,

$$
\operatorname{Re}\left\{\tilde{\mu}\left(-\omega+i 0^{+}\right)\right\}=\operatorname{Re}\left\{\tilde{\mu}\left(\omega+i 0^{+}\right)\right\} \geq 0 .
$$

Using the expression (1.3) for the correlation function, the mean square displacement can be written

$$
s(t)=\frac{2 \hbar}{\pi} \int_{0}^{\infty} d \omega \operatorname{Im}\left\{\alpha\left(\omega+i 0^{+}\right)\right\} \operatorname{coth} \frac{\hbar \omega}{2 k T}(1-\cos \omega t) .
$$

It is convenient to consider instead of $s$ its time derivative

$$
\dot{s}(t)=\frac{2 \hbar}{\pi} \int_{0}^{\infty} d \omega \operatorname{Im}\left\{\alpha\left(\omega+i 0^{+}\right)\right\} \omega \operatorname{coth} \frac{\hbar \omega}{2 k T} \sin \omega t .
$$

It should be clear from this formula that the long time behavior follows from the low frequency behavior of the integrand. We now show how this works for the most general case.

\section{OHMIC CASE.}

Consider first the Ohmic case, where $\tilde{\mu}(z)=\zeta$, a constant. Then

$$
\alpha\left(\omega+i 0^{+}\right)=\frac{1}{-m \omega^{2}-i \zeta \omega}
$$

and (1.7) becomes

$$
\dot{s}(t)=\frac{2 \hbar}{\pi} \int_{0}^{\infty} d \omega \frac{\zeta}{m^{2} \omega^{2}+\zeta^{2}} \operatorname{coth} \frac{\hbar \omega}{2 k T} \sin \omega t .
$$

The long time behavior is obtained by expanding the part of the integrand other than the $\sin \omega t$ function in powers of $\omega$. That is,

$$
\dot{s}(t) \sim \frac{4 k T}{\pi \zeta} \int_{0}^{\infty} d \omega \frac{\sin \omega t}{\omega}=\frac{2 k T}{\zeta} .
$$


Thus $\dot{s}$ is a constant and the mean square displacement is linear in time. This is the case of so-called normal diffusion.

The case of zero temperature requires that in (2.2) we set $\operatorname{coth} \frac{\hbar \omega}{2 k T} \rightarrow 1$ first and then expand in powers of $\omega$, to obtain

$$
\dot{s}(t) \sim \frac{2 \hbar}{\pi \zeta} \int_{0}^{\infty} d \omega \sin \omega t=\frac{2 \hbar}{\pi \zeta t} .
$$

Note that this is a quantum result, vanishing in the limit $\hbar \rightarrow 0$, while the finite temperature result (2.3) is classical. This is a general feature: diffusion (normal or anomalous) is classical at finite temperature, quantum at zero temperature. The fact that zero-temperature diffusion involves one power of $t$ less than for finite temperature is also general.

It will be of some interest to exhibit the exact zero-temperature result, which takes the form

where [13]

$$
\dot{s}(t)=\frac{2 \hbar}{\pi \zeta t} V\left(\frac{\zeta t}{m}\right)
$$

$$
V(x)=\int_{0}^{\infty} d u \frac{x^{2} \sin (u)}{x^{2}+u^{2}}=\frac{x}{2}\left[e^{-x} \overline{\operatorname{Ei}}(x)-e^{x} \operatorname{Ei}(-x)\right] .
$$

From its form, it should be clear that the asymptotic form of the mean square displacement at zero temperature is given by

$$
s(t) \sim \frac{2 \hbar}{\pi \zeta}\left(\log \frac{\zeta t}{m}+\gamma_{E}\right)
$$

where $\gamma_{E}=0.577 \cdots$ is Euler's constant.

\section{COLORED NOISE.}

Consider the case where in the neighborhood of the origin

$$
\tilde{\mu}(z)=m b^{1-\gamma}(-i z)^{\gamma}, \quad-1<\gamma<1,
$$

where $b$ is a constant with the dimensions of frequency. It is easy to verify that this is a positive real function if and only if $\gamma$ is within the indicated range (we choose the branch with $-\pi<\arg (z)<\pi)$. With this, we see that (1.7) becomes

$$
\dot{s}(t)=\frac{2 \hbar}{\pi} \int_{0}^{\infty} d \omega \frac{\cos \left(\frac{\pi}{2} \gamma\right) \operatorname{coth} \frac{\hbar \omega}{2 k T}}{m \omega\left[\left(\frac{\omega}{b}\right)^{1-\gamma}+\left(\frac{b}{\omega}\right)^{1-\gamma}+2 \sin \left(\frac{\pi}{2} \gamma\right)\right]} \sin \omega t .
$$


Expanding the integrand for small $\omega$, we find for large $t$,

$$
\dot{s}(t) \sim \frac{4 k T \cos \left(\frac{\pi}{2} \gamma\right)}{\pi m b^{1-\gamma}} \int_{0}^{\infty} d \omega \frac{\sin \omega t}{\omega^{\gamma+1}}
$$

Since

$$
\int_{0}^{\infty} d x \frac{\sin x}{x^{\gamma+1}}=\frac{\pi}{2 \Gamma(1+\gamma) \cos \left(\frac{\pi}{2} \gamma\right)}
$$

we have the result

$$
\dot{s}(t) \sim \frac{2 k T}{m b^{1-\gamma} \Gamma(1+\gamma)} t^{\gamma} .
$$

Thus, we see that colored noise corresponds to anomalous diffusion. In addition we see that there is a physical reason for the restricted range of $\gamma$ : it is a consequence of the second law of thermodynamics.

The case of zero temperature follows in the same way as in the Ohmic case. Setting $\operatorname{coth} \frac{\hbar \omega}{2 k T} \rightarrow 1$ and expanding in powers of $\omega$, (3.2) becomes

$$
\dot{s}(t) \sim \frac{2 \hbar \cos \left(\frac{\pi}{2} \gamma\right)}{\pi m b^{1-\gamma}} \int_{0}^{\infty} d \omega \frac{\sin \omega t}{\omega^{\gamma}}=\frac{\hbar \cot \left(\gamma \frac{\pi}{2}\right)}{\Gamma(\gamma) m b^{1-\gamma}} t^{\gamma-1} .
$$

Note that the case $\gamma=0$ is not singular $\left(\lim _{\gamma \rightarrow 0} \cot \left(\frac{\pi}{2} \gamma\right) / \Gamma(\gamma)=2 / \pi\right)$. Note also that this zero-temperature result involves one power of $t$ less than the finite temperature result (3.5).

The extremes of the range of $\gamma, \gamma= \pm 1$, require special attention. The case $\gamma=-1$ corresponds to a generalized susceptibility of the form

$$
\alpha(z)=\frac{1}{-m z^{2}+m b^{2}} .
$$

This form corresponds to a free oscillator with force constant $K=m b^{2}$. That is, $\gamma=-1$ corresponds to a harmonically bound particle. For this case one can obtain from (1.7) the exact result

$$
\dot{s}(t)=\frac{\hbar}{m} \operatorname{coth} \frac{\hbar b}{2 k T} \sin b t .
$$

Thus, with $\gamma=-1$ the particle is bound and there is no diffusion. This is consistent with (3.5), for which the multiplier vanishes for $\gamma=-1$.

The case $\gamma=+1$ in (3.1) clearly corresponds to a shift in the particle mass, with no dissipation. In the absence of dissipation, it should be obvious that at finite temperature the mean square displacement should grow with the square of the time, consistent with (3.5) for this case. There is, however, a non-trivial example for which dissipation is present and the mean square displacement at finite temperature nevertheless grows as the square of the time. This is the quantum electrodynamic (QED) case, which we discuss next. 


\section{QED CASE.}

In the case of a nonrelativistic electron coupled to the radiation field, the Fourier transform of the memory function in QED can be written in the form: 14, 15]

$$
\tilde{\mu}(z)=\frac{2 e^{2}}{3 c^{3}} \frac{z \Omega^{2}}{z+i \Omega},
$$

where $\Omega$ is a large cut-off frequency, related to the electron form-factor. With this, the form (1.4) for the generalized susceptibility for a free particle becomes

$$
\alpha(z)=\frac{z+i \Omega}{-m z^{3}-i M \Omega z^{2}}
$$

where $m$ is the bare mass and

$$
M=m+\frac{2 e^{2} \Omega}{3 c^{3}}
$$

is the renormalized (physical) mass. We can use $m$ as a parameter in place of $\Omega$, writing

$$
\Omega=\frac{M-m}{M \tau_{e}}, \quad 0 \leq m \leq M,
$$

where

$$
\tau_{e}=\frac{2 e^{2}}{3 M c^{3}} \cong 6.25 \times 10^{-24} \mathrm{~s} .
$$

With this, we can write the generalized susceptibility in the form

$$
\alpha(z)=\frac{M-m-i M z \tau_{e}}{-M z^{2}\left(M-m-i m z \tau_{e}\right)} .
$$

Note that with this, $m$ can be viewed as a measure of the strength of coupling, with $0 \leq$ $m \leq M$. The limit $m=M$ corresponds to no interaction (i.e., free particle) and the limit $m=0$ corresponds maximal coupling, where the cut-off has its largest value consistent with causality $\left(\Omega=\tau_{e}^{-1}\right)$.

We can rearrange the form (4.6) to write

$$
\alpha(z)=-\frac{1}{M z^{2}}+\frac{(M-m) \tau_{e}}{-i M z\left(M-m-i m z \tau_{e}\right)} .
$$

Then we see that

$$
\operatorname{Im}\left\{\alpha\left(\omega+i 0^{+}\right)\right\}=-\frac{\pi}{M} \delta^{\prime}(\omega)+\frac{(M-m)^{2} \tau_{e}}{M \omega\left[(M-m)^{2}+\left(m \omega \tau_{e}\right)^{2}\right]},
$$

where $\delta^{\prime}$ is the derivative of the delta-function. With this, the expression (1.7) for $\dot{s}$ becomes

$$
\dot{s}(t)=\frac{2 k T}{M} t+\frac{2 \hbar \tau_{e}}{\pi M} \int_{0}^{\infty} d \omega \frac{(M-m)^{2}}{(M-m)^{2}+\left(m \omega \tau_{e}\right)^{2}} \operatorname{coth} \frac{\hbar \omega}{2 k T} \sin \omega t .
$$




\section{A. Free particle.}

Consider first the case of a free particle, with no interaction with the radiation field. In this case only the first term in the expression (4.9) survives, and we have the result,

$$
s(t)=\frac{k T}{M} t^{2}, \quad \text { free particle }
$$

valid for all times. This result follows from equipartition $\left(\left\langle\frac{1}{2} M v^{2}\right\rangle=\frac{1}{2} k T\right)$ which for a free particle is equally true in classical and quantum mechanics. Note that this is an exact result: for a free particle at zero temperature $s(t)=0$ for all time.

\section{B. Maximal coupling.}

Next consider the case of maximal coupling, where $m=0$. In this case we find the exact closed form result [13],

$$
\begin{aligned}
\dot{s}(t) & =\frac{2 k T}{M} t+\frac{2 \hbar \tau_{e}}{\pi M} \int_{0}^{\infty} d \omega \operatorname{coth} \frac{\hbar \omega}{2 k T} \sin \omega t \\
& =\frac{2 k T}{M}\left(t+\tau_{e} \operatorname{coth} \frac{\pi k T t}{\hbar}\right) .
\end{aligned}
$$

Thus, for long time $\dot{s}$ grows linearly with time. Indeed the mean square displacement for long times is exactly of the form (4.10) for a free particle. This is what we should expect since in QED a particle moving with constant velocity feels no force.

At zero temperature, the exact expression (4.11) becomes

$$
\dot{s}(t)=\frac{2 \hbar \tau_{e}}{\pi M} \frac{1}{t} .
$$

Note that for long times this result is an exception to the general rule that for colored noise the long time dependence at zero temperature involves one power of $t$ less than at finite temperature. Here the difference is two powers of $t$. This phenomenon is already signalled in the general expression (3.6) for colored noise at zero temperature, where the multiplier vanishes for $\gamma=1$.

We note that at short times the exact expression (4.11) diverges like $t^{-1}$. This should be puzzling, since by it's very definition (1.1) $s(0)=0$. This divergence is a quantum phenomenon, arising from the mass renormalization. In order to understand this, we consider next the general expression (4.9) at zero temperature. 


\section{General case at zero temperature.}

In the limit of zero temperature, the general expression (4.9) becomes

$$
\dot{s}(t)=\frac{2 \hbar \tau_{e}}{\pi M} \int_{0}^{\infty} d \omega \frac{(M-m)^{2}}{(M-m)^{2}+\left(m \omega \tau_{e}\right)^{2}} \sin \omega t .
$$

For long times this vanishes like $t^{-1}$ (at zero temperature the particle is at rest). For very short times, of order $m \tau_{e} / M$, this increases rapidly from zero to a large value, after which it falls to zero again like $t^{-1}$. In fact, this integral is exactly that appearing in the corresponding Ohmic case (2.5). We can therefore write

$$
\dot{s}(t)=\frac{2 \hbar \tau_{e}}{\pi M t} V\left(\frac{(M-m) t}{m \tau_{e}}\right),
$$

where $V(x)$ is given by (2.6). This is an example in which the bare mass appears for extremely short times (i.e., extremely high frequencies) Another example is the canonical commutation rule [16]. Clearly, in the limit $m \rightarrow 0$, (4.14) becomes exactly of the form

(4.12), but now with $\frac{1}{t}$ interpreted as the principal value. As in the Ohmic case, in order to integrate to find the asymptotic form of the mean square displacement we need to know the behavior at small $t$. With the result (4.14) we see that

$$
s(t) \sim \frac{2 \hbar \tau_{e}}{\pi M} \log \frac{(M-m) t}{m \tau_{e}}+\gamma_{E} .
$$

Thus, we see that the bare mass appears even in the long time behavior of $s(t)$. We shall have more to say about this in our discussion of wave packet spreading.

\section{SPREADING OF A WAVE PACKET.}

As a somewhat different application of these results, we consider the spreading of a Gaussian wave packet. The situation is as follows. At time $t_{1}$ the Brownian particle, which before the measurement is in equilibrium at temperature $T$, is measured (i.e., detected) with a Gaussian instrument of width $\sigma_{1}$ and centered at $x_{1}$. Then at a later time $t_{2}$ a second measurement is made, again with a Gaussian instrument but now with width $\sigma_{2}$ and centered at $x_{2}$. Let $W(1,2) d x_{1} d x_{2}$ be the probability that the first measurement finds the particle in range $d x_{1}$ about $x_{1}$ and that the second measurement finds the particle in range $d x_{2}$ about $x_{2}$. The mean square width of the wave packet at time $t_{2}$ is then given by

$$
w^{2}\left(t_{2}-t_{2}\right) \equiv \int_{-\infty}^{\infty} d x_{1} \int_{-\infty}^{\infty} d x_{2} W(1,2)\left(x_{1}-x_{2}\right)^{2} .
$$


Here we must be sure to distinguish this expectation from that in (1.1); here $x_{1}$ and $x_{2}$ are instrumental parameters (i.e., c-numbers) not quantum mechanical operators. One could well argue that for a quantum particle this wave packet width $w^{2}$ is a better measure of anomalous diffusion than the mean square displacement $s$. The picture, to repeat, is the following. An initial wave packet of width $\sigma_{1}$ is formed at time $t_{1}$. In the course of time this wave packet will spread. It's center will not move since the mean velocity is zero. At a later time $t_{2}$ the mean square width, including the width $\sigma_{2}$ of the second instrument, is given by (5.1).

Now, for quantum Brownian motion an exact expression for $W(1,2)$ can be obtained [17],

$$
W(1,2)=\frac{1}{2 \pi \sigma \tau \sqrt{1-\rho^{2}}} \exp \left\{-\frac{1}{2\left(1-\rho^{2}\right)}\left(\frac{x_{1}^{2}}{\sigma^{2}}-2 \frac{\rho x_{1} x_{2}}{\sigma \tau}+\frac{x_{2}^{2}}{\tau^{2}}\right)\right\}
$$

where (note a misprint in Eq. 7.18 of [17])

$$
\begin{aligned}
\sigma^{2} & =\sigma_{1}^{2}+\left\langle x\left(t_{1}\right)^{2}\right\rangle \\
\tau^{2} & =\sigma_{2}^{2}+\left\langle x\left(t_{1}\right)^{2}\right\rangle-\frac{\left[x\left(t_{1}\right), x\left(t_{2}\right)\right]^{2}}{4 \sigma_{1}^{2}} \\
\sigma \tau \rho & =\frac{1}{2}\left\langle x\left(t_{1}\right) x\left(t_{2}\right)+x\left(t_{2}\right) x\left(t_{1}\right)\right\rangle .
\end{aligned}
$$

With this, we find that (5.1) becomes [18]

$$
w^{2}\left(t_{2}-t_{1}\right)=s\left(t_{2}-t_{1}\right)+\sigma_{1}^{2}-\frac{\left[x\left(t_{1}\right), x\left(t_{2}\right)\right]^{2}}{4 \sigma_{1}^{2}}+\sigma_{2}^{2} .
$$

Thus, the mean square width of the wave packet is a sum of four terms. The first is the mean square displacement (1.1). The second and third terms involve the finite width of the initial wave packet, with the third term clearly arising from the uncertainty principle. Finally, the last term is the mean square width of the second instrument. Clearly, this final width can be taken to be zero, but not the initial width.

For quantum Brownian motion, the commutator is a c-number given by 12 ]

$$
\left[x\left(t_{1}\right), x\left(t_{2}\right)\right]=\frac{2 i \hbar}{\pi} \int_{0}^{\infty} d \omega \operatorname{Im}\left\{\alpha\left(\omega+i 0^{+}\right)\right\} \sin \omega\left(t_{2}-t_{1}\right) .
$$

Note that the commutator is temperature independent. We now consider a number of cases.

\section{A. Colored noise}

For long times, using the method described above, we find for colored noise,

$$
\left[x\left(t_{1}\right), x\left(t_{2}\right)\right] \sim \frac{i \hbar}{m b^{1-\gamma} \Gamma(1+\gamma)} t^{\gamma}
$$


where to save writing we have written $t=t_{2}-t_{1}$. At finite temperature, using this result for the commutator and the corresponding result (3.5) for the mean square displacement, we see that for $-1<\gamma<1$ and for sufficiently long times the term $s\left(t_{2}-t_{1}\right)$ dominates in (5.4). Thus at finite temperature, the long time behavior for colored noise is the same, whether measured by the mean square displacement (1.1) or by the wave packet width (5.1), and always classical.

On the other hand, at zero temperature, using the result (3.6) we see that the general expression (5.4) becomes

$$
\begin{aligned}
w^{2}(t) \sim & \frac{\hbar \cot \left(\gamma \frac{\pi}{2}\right)}{\Gamma(1+\gamma) m b^{1-\gamma}} t^{\gamma} \\
& +\sigma_{1}^{2}+\frac{\hbar^{2}}{4 m^{2} \sigma_{1}^{2} b^{2-2 \gamma} \Gamma(1+\gamma)^{2}} t^{2 \gamma}+\sigma_{2}^{2}
\end{aligned}
$$

Here the first term corresponds to $s(t)$ and we see that for $-1<\gamma<0$ this term still dominates at zero temperature. The Ohmic case $\gamma=0$ appears to be singular, but as we noted above this is not a problem. In fact, for the Ohmic case at zero temperature we have

$$
w_{\text {Ohmic }}^{2}(t) \sim \frac{2 \hbar}{\pi \zeta} \log \frac{\zeta t}{m}+\sigma_{1}^{2}+\frac{\hbar^{2}}{4 \sigma_{1}^{2} \zeta^{2}}+\sigma_{2}^{2} .
$$

Thus, in the Ohmic case, we see that the first term still dominates. On the other hand, for $0<\gamma<1$, for sufficiently long times the third term dominates, corresponding to what we might call uncertainty principle spreading.

\section{B. Free particle}

For the free particle, using the expression (4.8) with $m=M$, the expression (5.5) for the commutator becomes

$$
\left[x\left(t_{1}\right), x\left(t_{2}\right)\right]=\frac{i \hbar}{M}\left(t_{2}-t_{1}\right) .
$$

With this and the expression (4.10) for the mean square displacement, we see that the general expression (5.4) becomes

$$
w_{\text {free }}^{2}(t)=\frac{k T}{M} t^{2}+\sigma_{1}^{2}+\frac{\hbar^{2} t^{2}}{4 M^{2} \sigma_{1}^{2}}+\sigma_{2}^{2} .
$$

First of all, we note that at zero temperature and with $\sigma_{2}^{2}=0$ this becomes exactly the formula for wave packet spreading found in textbooks [19]. For finite temperature the 
first term, the mean square displacement, has the same $t^{2}$ dependence as the third term, the uncertainty principle spreading. The ratio of these two terms is $\lambda^{2} / 8 \pi \sigma_{1}^{2}$, where $\lambda=$ $\sqrt{2 \pi \hbar^{2} / M k T}$ is the mean thermal de Broglie wavelength. Thus, we see that the spreading will be classical provided that $\lambda \ll \sigma_{1}$.

\section{QED}

As in our discussion of colored noise, the case $\gamma=1$ requires special consideration and we discuss it in terms of the QED case. For the general form (4.8) of $\operatorname{Im}\{\alpha\}$, we see that (5.5) becomes

$$
\left[x\left(t_{1}\right), x\left(t_{2}\right)\right]_{\mathrm{QED}}=\frac{i \hbar}{M}\left\{t+\frac{2}{\pi} \int_{0}^{\infty} d \omega \frac{(M-m)^{2} \tau_{e} \sin \omega t}{\omega\left[(M-m)^{2}+\left(m \omega \tau_{e}\right)^{2}\right]}\right\} .
$$

The integral appearing here is standard [13], and we find

$$
\left[x\left(t_{1}\right), x\left(t_{2}\right)\right]_{\mathrm{QED}}=\frac{i \hbar}{M}\left\{t+\tau_{e}\left(1-e^{-(M-m) t / m \tau_{e}}\right)\right\} .
$$

Here the exponential is exceedingly small for any time of the order of $\tau_{e}$ or longer. It does, however, guarantee that the canonical equal-time commutator, $[x(t), \dot{x}(t)]_{\mathrm{QED}}=i \hbar / m$, does involve the bare mass, as it must [16].

With this result, it should be clear that for long times the leading behavior of the wave packet width in QED is the same as that for a free particle. There will be lower order corrections, of course, but these are perhaps best discussed in terms of the zero temperature result. At zero temperature, using the results (4.15) and (5.12) we find

$$
w_{\mathrm{QED}}^{2}(t) \sim \frac{2 \hbar \tau_{e}}{\pi M}\left\{\log \frac{(M-m) t}{m \tau_{e}}+\gamma_{E}\right\}+\sigma_{1}^{2}+\frac{\hbar^{2}\left(t+\tau_{e}\right)^{2}}{4 M^{2} \sigma_{1}^{2}}+\sigma_{2}^{2} .
$$

\section{CONCLUSIONS}

The first calculation of normal diffusion [equivalent to our equation (2.3)] was carried out by Einstein in his famous explanation [20] for the observations of Brown 21] on the random motion of pollen grains immersed in a fluid. The term "Brownian motion" is now used in a generic sense to denote random motion and it covers a wide spectrum of phenomena. Einstein's explanation of the original Brownian motion used a diffusion equation but, shortly afterwards, Langevin [22] presented an entirely new approach to the subject, based on the 
introduction of a stochastic differential equation, which in the words of Chandrasekhar [23], constitutes the "modern" approach to this and other such problems. Langevin's analysis was confined to the classical long-time motion of a free particle at high temperature in an Ohmic heat bath (based on Stokes analysis of viscous drag).

Here, we use a generalized Langevin equation which incorporates quantum and nonMarkovian (memory) effects, arbitrary times, arbitrary temperature and a very general heat bath. This leads to a fluctuation-dissipation theorem which enables us to calculate anomalous (in a sense that it goes beyond the Einstein-Langevin case) diffusion for a wide variety of parameters which, in turn, will facilitate analysis of a variety of physical phenomena. In particular, we applied our results to wave-packet spreading. We consider the use of the quantum Langevin equation and the fluctuation-dissipation theorem to be simpler and more physically appealing than approaches based on path-integral techniques [9, 24]. In addition, our description of the motion of a quantum particle in a linear passive heat bath is characterized by $\operatorname{Re}[\tilde{\mu}(z)]$, the Fourier transform (spectral distribution) of the memory function appearing in the quantum Langevin equation. As a consequence of the second law of thermodynamics, $\tilde{\mu}(z)$ must be a positive real function [12], that is $\tilde{\mu}(z)$ must be analytic with positive real part everywhere in the upper half plane and, in addition, its boundary value on the real axis, which in general may be a distribution, must satisfy the reality condition, given by (1.5). As a consequence, this limits the forms of $\tilde{\mu}(z)$, as exemplified in (3.1), which

in turn limits the forms of anomalous diffusion. Finally, we discussed in detail the special case of an electron interacting with the radiation field.

\section{Acknowledgments}

We wish to thank the School of Theoretical Physics, Dublin Institute for Advanced Studies, for their hospitality.

[1] J. Klafter and I.M. Sokolov, Phys. World Aug. 2005, p.29.

[2] M.F. de Andrade et al., Phys. Lett. A 347, 160 (2005).

[3] E. Lutz, Phys. Rev. E 64, 051106 (2001).

[4] C. Tsallis and D.J. Bukman, Phys. Rev. E 54, R2197 (1996). 
[5] M. O. Scully, G. Sussmann and C. Benkert, Phys. Rev. Lett. 60, 1014 (1988)

[6] M. O. Scully, M. S. Zubairy and K. Wodkiewicz, Optics Comm. 65, 440 (1988)

[7] C. Benkert, M.O. Scully, A.A. Rangwala and W. Schleich, Phys. Rev. A 43, 1503 (1990).

[8] S. Schaufler, W.P. Schleich and V.P. Yakovlev, Phys. Rev. Lett. 83, 3162 (1999).

[9] H. Grabert, P. Schramm and G.-L. Ingold, Phys. Rev. Lett. 58, 1285 (1987).

[10] H.B. Callen and T.A. Welton, Phys. Rev. 83, 34 (1951).

[11] G.W. Ford, J.T. Lewis and R.F. O'Connell, Ann. Phys. (NY) 185, 270 (1988).

[12] G.W. Ford, J.T. Lewis and R.F. O’Connell, Phys. Rev. A 37, 4419 (1988).

[13] Tables of integral transforms, edited by Staff of the Bateman Manuscript Project, Bateman manuscript project, Vol. 1 (McGraw-Hill, New York, 1954).

[14] G.W. Ford, J.T. Lewis and R.F. O'Connell, Phys. Rev. Lett. 55, 2273 (1985).

[15] G.W. Ford and R.F. O’Connell, Phys. Rev. A 57, 3112 (1998).

[16] G.W. Ford and R.F. O'Connell, Journ. Stat. Phys. 57 (1989) 803.

[17] G. W. Ford and J. T. Lewis, Probability, Statistical Mechanics, and Number Theory, Advances in Mathematics Supplemental Studies, Volume 9 (Academic Press, Orlando, Florida 1986)

[18] G.W. Ford, J.T. Lewis and R.F. O’Connell, Phys. Rev. A 64, 032101 (2001).

[19] L. I. Schiff, Quantum Mechanics (McGraw-Hill, New York 1949) p. 58.

[20] A. Einstein, Eine neue Bestimmung der Moleküldimensionen, (Wyss, Bern, 1905) [Einstein's thesis]; ibid. Ann. d. Physik 17, 549 (1905); this and the further articles of Einstein appear in Investigations on the Theory of the Brownian Movement (Dover, New York, 1956).

[21] R. Brown, Philos. Mag. 4, 161 (1828); Ibid. 6, 161 (1829).

[22] M. P. Langevin, C. R. Acad, Sci., Paris 146, 530 (1908) (a translation of this article appears in D.S. Lemons, A. Gythiel, Am. J. Phys. 651079 (1997)).

[23] S. Chandrasekhar, Rev. Mod. Phys. 15, 1 (1943).

[24] V. Hakim and V. Ambegaokar, Phys. Rev. A 32, 423 (1985). So far as we know, this paper contains the first derivation of the width of a spreading wave packet, equivalent with our Eq. (5.4), but only for the ohmic case. Also, we note a misprint in their expression (38) for the width. 